\title{
Kernos
}

Revue internationale et pluridisciplinaire de religion grecque antique

$2 \mid 1989$

Varia

\section{Anytos, le parèdre armé de Despoina à Lykosoura}

\author{
Éveline Loucas-Durie
}

URL : http://journals.openedition.org/kernos/243

DOI : $10.4000 /$ kernos. 243

ISSN : 2034-7871

\section{Éditeur}

Centre international d'étude de la religion grecque antique

\section{Édition imprimée}

Date de publication : 1 janvier 1989

Pagination : 105-114

ISSN : 0776-3824

Référence électronique

Éveline Loucas-Durie, «Anytos, le parèdre armé de Despoina à Lykosoura », Kernos [En ligne], 2 | 1989, mis en ligne le 02 mars 2011, consulté le 19 avril 2019. URL : http://journals.openedition.org/ kernos/243; DOI : 10.4000/kernos.243 
Kernos, 2 (1989), p. 105-114.

\section{ANYTOS, LE PARÈDRE ARMÉ DE DESPOINA À LYKOSOURA*}

Le sanctuaire de Lykosoura, qui a déjà plus d'une fois attiré notre curiosité ${ }^{1}$, était consacré à cette déesse honorée par un rite orgiastique et appelée Despoina; elle était considérée comme la fille de Déméter et de Poseidon hippios ${ }^{2}$, et Damophon de Messène l'avait figurée assise sur un trône aux côtés de sa mère ${ }^{3}$. Ce couple de déesses était flanqué à gauche et à droite d'une statue de parèdre debout. Celle qui était située près de Despoina montrait un homme en armes ${ }^{4}$. Plus précisément, d'après les fragments retrouvés et la représentation figurant au revers d'une monnaie de Mégalopolis frappée à l'image de Julia Domna, dans laquelle on reconnaît généralement le groupe ${ }^{5}$, il était revêtu d'une cuirasse à lambrequins frangés et tenait dans la main gauche levée une lance sur laquelle il prenait vraisemblablement appui ${ }^{6}$. Son nom ? Seul Pausanias, qui a vu et décrit l'ensemble des quatre statues, nous l'apprend : Anytos. Et le Périégète ajoute

* Le point de départ de cet article est le chapitre intitulé Anytos de mon mémoire de licence Lykosoura, lieu de culte chthonien, Université de Bruxelles, 1984. Toutefois, la figure d'Anytos est ici envisagée d'un point de vue différent.

1 É. (LOUCAS-) DURIE, Les fonctions sacerdotales au sanctuaire de Despoina à Lykosoura, in Horos, 2 (1984), p. 137-148; É. LouCAs, Le nom de la Thea Despoina (Tatien, Ad Graec., 29 - Paus., VIII, 37.6-9), Actes du 3e colloque international des études péloponnésiennes, Kalamata, 8-15/9/1985, in Peloponnesiaka, suppl. 13 (19871988), t. 2, p. 401-419; I. LOUCAS et É. LOUCAS-DURIE, La mention du mot «agalma» dans la loi sacrée de Lykosoura IG, V 2, 514, in Peloponnesiaka, 16 (1985-1986), p. 561-578; I. et É. LoUCAS, The Despoina Megaron at Lykosoura and Certain Prehistoric Telesteria, in JPR, 2 (1988) (sous presse).

2 PAUS., VIII, 37, 9; 42, 1.

3 PAUS., VII, 37, 2-4. À propos de ce passage, cf. Le nom de la Thea Despoina..., p. 418 et n. 115. Pour Damophon et le groupe statuaire cultuel de Lykosoura, cf. la présentation générale et récente de M. JosT, Sanctuaires et cultes d'Arcadie, Paris, 1985 (Études péloponnésiennes, IX), p. 175-176, 327-328; E. LÉvY-J. MARCADÉ, $A u$ Musée de Lykosoura, in BCH, 96 (1972), p. 967-1004; cf. aussi E. LÉVY, Sondages à Lykosoura et date de Damophon, in BCH, 91 (1967), p. 518-545, où l'on trouvera la bibliographie antérieure.

4 PAUS., VIII, 37, 5.

5 B. STAIS, in JLAN, 14 (1912), p. 45-47. Cf. LÉVY \& MARCADÉ, art. cit., p. 989 et fig. 41 p. 1003.

6 LÉVY \& MARCADÉ, art. cit., p. 988-991. 
que les gens des alentours du sanctuaire disaient qu'il avait élevé Despoina et devait être compté parmi les Titans?.

Le nom d'Anytos semble tout indiqué pour un père nourricier. Ce serait sans doute encore ce rôle qu'un moderne, exploitant les ressources de l'étymologie, aurait attribué au personnage, inconnu par ailleurs si ce n'est par un anthroponyme Anuto sur une tablette de Pylos ${ }^{8}$. En outre, son apparence armée l'inscrit dans une lignée de personnages de nature non humaine qui ont porté secours à une divinité dotée d'une certaine importance, ne fût-ce que dans un panthéon local. Ainsi Anytos a été rapproché d'Hoplodamos (son nom semble indiquer un lien direct avec les armes) et de ses Géants qui, selon les habitants de Méthydrion, s'étaient engagés envers Rhéa, enceinte de Zeus et venue se cacher au mont Thaumasion, à intervenir si Kronos arrivait ${ }^{9}$. Mais la tradition arcadienne disait que c'était finalement sur le mont Lykaon que Rhéa avait mis Zeus au monde, plus précisément dans une région du mont appelée Kretea où le nouveau-né passa les premières années de son existence ${ }^{10}$. L'Arcadie disputait en effet l'honneur d'être le berceau de Zeus à la Crète où, selon l'une des nombreuses versions du mythe, le dieu aurait grandi sous la bienveillante protection des Dactyles ou des Courètes.

Les Dactyles de l'Ida, suivant l'origine crétoise ou phrygienne que leur donnaient les Anciens, paraissent avoir d'abord été les acolytes de Rhéa ou de Cybèle, maîtres en l'art des conjurations, des rites, des mystères et, métallurgistes accomplis, en celui du travail du bronze et du fer ${ }^{11}$. Héraclès, qui organisa pour la première fois les Olympia, aurait même été un des leurs, rapporte Pausanias ${ }^{12}$, mais le Périégète précise ailleurs que telle est l'opinion qu'Onomacrite fit valoir dans ses poèmes à propos d'une statue du héros haute d'une coudée qui s'élevait à côté de celle de Déméter dans le sanctuaire des Grandes Déesses à Mégalopolis ${ }^{13}$.

7 PAUS., VIII, $37,5$.

8 Cf. P. Chantraine, Dictionnaire étymologique de la langue grecque, Paris, I. A - $\Delta$,

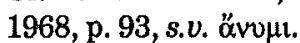

9 PAUS., VIII, 36, 2-3. Cf. F. VIAN, La guerre des Géants. Le mythe avant l'époque hellénistique, Paris, 1952 (Études et commentaires, XI), p. 240; JosT, op. cit., p. 335.

10 PAUS., VIII, 36, 3; 38, 2.

11 Diod. Sic., V, 65. Cf. H. JEANMaIRE, Couroi et Couretes, Lille, 1939, p. 439.

12 PAus., V, 7, 6 et 9. Cf. aussi V, 14, 9.

13 PAUS., VIII, 31, 3. 
Au témoignage des Anciens eux-mêmes ${ }^{14}$, les Dactyles furent confondus avec les Courètes et c'était comme tels qu'ils étaient vénérés à Olympie ${ }^{15}$. Les Courètes auraient enseigné aux hommes la chasse, le tir à l'arc, l'apprivoisement des troupeaux, l'élevage des abeilles, c'est-à-dire des techniques grâce auxquelles l'être humain peut désormais s'affirmer dans la nature et en régulariser un tant soit peu à son profit le cycle parfois irrégulier ${ }^{16}$. Les Courètes étaient encore tenus pour inventeurs des armes et de la danse armée et, selon la légende, ils avaient étouffé les vagissements de Zeus nouveau-né tandis qu'ils évoluaient en dansant autour de l'enfant divin en entrechoquant leurs boucliers et leurs épées ${ }^{17}$.

Une tradition qui avait cours dans cette région limitrophe de l'Élide et de l'Arcadie qu'est la Messénie rattachait les Courètes aux enfances «locales» de Zeus ${ }^{18}$ et il y avait à Messène, selon Pausanias, un "megaron" des Courètes où étaient sacrifiées dans les flammes toutes sortes d'animaux ${ }^{19}$. À Lykosoura, où il y avait également un «megaron" (une sorte d'autel monumental consacré à Despoina, construit à flanc de colline, où était célébrée dans une atmosphère orgiastique la télétè en son honneur) ${ }^{20}$, le Périégète signale au pied du groupe cultuel des représentations de Courètes (peut-être sous les statues d'Artémis et d'Anytos), voisinant avec celles de Corybantes sur la base du trône ${ }^{21}$. De toute cette ornementation, il ne reste aujourd'hui qu'une plaque qui décorait la face latérale du marche-pied des deux déesses avec un motif de fauves de part et d'autre d'un tympanon ${ }^{22}$.

L'apparente similitude de fonction et d'attributs entre ces personnages et le père nourricier armé de Despoina a conduit $F$. Vian et $M$. Jost à reconnaître en Anytos "le prolongement de l'un de ces démons armés qui entouraient la Déesse Mère préhellénique» et en étaient les serviteurs et

14 Cf., par ex., STRAB., X, 3, 22; PAUS., V, 7, 6; cf. aussi V, 14, 9.

15 PAUS., V, 8, 1.

16 Diod. Sic., V, 65. Cf. JEANMAIRE, op. cit., p. 438.

17 Strab., X, 480; Apoll., Bibl., I, 1, 6-7.

18 PAUS., IV, 33, 1.

19 PAUS., IV, $31,9$.

20 PAUS., VIII, 37, 8. Cf. pour les vestiges K. KourouniotIs, in $A E$ (1912), p. 142-161; I. \& É. LouCAS, The Megaron at Lykosoura ...; pour la téléte, LOUCAS \& LoUCAS-DURIE, $L a$ mention du mot «agalma»...

21 PAUS., VIII, 37, 6. Pour une autre proposition de la distribution du décor, cf. JOST, op. cit., p. 328.

22 LEVY \& MARCADE, art. cit., p. 999-1002; JOST, op. cit., p. 328. 
courotrophes ${ }^{23}$. Il est notable que, parmi ses «semblables" plus ou moins absorbés dans le cycle de Zeus, Anytos serait le seul à faire état d'une parenté directe et aussi nette avec la Grande Déesse ${ }^{24}$, dont le souvenir aurait donc été perpétué par Despoina, en vertu de sa prépondérance incontestable sur Déméter dans tout le sanctuaire, et des traces vivaces de thériomorphisme et de sauvagerie qui lui sont associées dans le culte. En tout cas, Anytos semble avoir bien rempli son rôle de protecteur auprès de la déesse, car, dans les autres localités arcadiennes où l'on connaît l'existence de cette fille de Déméter et de Poseidon hippios, et où, en même temps, on ignore celle d'Anytos, Despoina n'est qu'une pâle figure légendaire dont on parvient à peine à reconstituer la genèse : tout au plus retire-t-on des témoignages des habitants de Thelpousa, Onkeio et Phigalie, qui ne coïncident pas parfaitement, que Despoina naquit des violences infligées par Poseidon hippomorphe à Déméter qui s'était momentanément métamorphosée en cavale dans le vain espoir d'échapper à son soupirant, tandis qu'elle errait à la recherche de sa fille ravie par Hadès 25 . Or, à Lykosoura, il n'est pas fait mention de la légende. Ce n'est qu'au fil du commentaire du Périégète que le lecteur apprend sèchement l'ascendance de Despoina.

La qualité de Titan attribué à Anytos par les Arcadiens eux-mêmes est loin d'apporter quelque lumière sur le personnage. Faut-il la comprendre en référence à la théogonie hésiodique et voir en Anytos un de ces candidats à la souveraineté entrés en compétition avec Zeus, mais vaincus par le chef des Olympiens parce qu'ils incarnent le désordre et l'Hubris ${ }^{26}$ ? Le terme Titan a-t-il d'ailleurs toujours recouvert le même «signifié» au cours du temps ? D'autre part, Pausanias suggère une relation, qui demeure, pour nous modernes, imprécise, entre Anytos et ces Titans qu'Onomacrite transforma en artisans de la passion de Dionysos ${ }^{27}$, divinité totalement inexistante à Lykosoura. Un pareil Titan n'est pas en soi déplacé dans le contexte cultuel

23 JOST, op. cit., p. 335-336; VIAN, op. cit., p. 240. M. JOST (op. cit., p. 245) avait mis l'accent avec raison sur une différence de fonction entre les Courètes, Hoplodamos et ses Géants. Les premiers veillaient sur Zeus, les seconds sur Rhéa.

24 Même si Hoplodamos et ses Géants doivent être identifiés à ceux qu'a vaincus Zeus à Bathos (PAUS., VIII, 29, 1; cf. VIAN, op. cit., p. 243-246), les Géants de Bathos ne sont pas associés aux Grandes Déesses autrement qu'au plan géographique et cultuel (PAUS., VIII, 29, 1). Cf. aussi infra.

25 PAUS., VIII, 25, 4-8; 42, 1-2. Cf. à propos de ces deux versions du mythe arcadien, I. LoUCAS, Le comportement de la déesse de la prospérité dans les mythes mésopotamiens et égéens de la Descente aux Enfers, in RHR, 205 (1988), p. 227-244.

26 Cf. J.-P. VeRnanT, Mythe et Pensée chez les Grecs, Paris, 1985, nouvelle édition revue et augmentée, p. 30.

27 PAUS., VIII, 37, 5. 
de Lykosoura, puisque les fidèles déguisés en animaux offraient à Despoina toutes sortes d'animaux sacrifiés par diasparagmos ${ }^{28}$, peut-être en lointain souvenir d'une victime humaine ${ }^{29}$. Ceci est cependant étrange, car Anytos était apparu au travers de sa fonction comme un être bienveillant, incarnant le courotrophe jusque dans son nom même. Cet apparent paradoxe pourrait recevoir un jour une réponse, mais qui exigerait qu'on étende le champ de recherche à des contextes parallèles faisant encore l'objet d'investigations qui dépassent le cadre de cette étude.

Dans le second volet de celle-ci que j'ouvre à présent, j'invite le lecteur à envisager l'Anytos de Lykosoura, non plus seulement pour ce qu'il était ou n'était pas dans l'univers des dieux, mais aussi pour ce qu'il pouvait être parmi les hommes - ceci dit sans anticiper sur une conclusion qui n'existe pas. Avec les informations déjà rassemblées, que peut-on imaginer aujourd'hui de ce qu'a pu signifier, pour un visiteur quelque peu averti, cette effigie d'homme armé à côté de Despoina?

Les figures mythiques comme les Géants de Thaumasion et les Courètes, auxquelles a pu être comparé Anytos en raison d'une analogie de fonction (la courotrophie) mais surtout d'attributs (les armes) semblent avoir eu également une «consistance» dans la vie des membres de la communauté humaine à une époque donnée.

Retrouvés à Palaiokastro, des fragments d'une copie du IIe ou IIIe s. ap. J.-C. d'un hymne plus ancien attestent des danses en l'honneur de Zeus appelé «très grand Kouros» ${ }^{30}$. Un Zeus hoplosmios était vénéré à Méthydrion même ${ }^{31}$, sous cette épiclèse attribuée ailleurs à Héra et peut-être aussi à Athéna $^{32}$. Et Hoplodmia, c'est le nom d'une tribu à Mantinée ${ }^{33}$, ville qui a laissé son nom à une danse, la mantinikè orchèsis, exécutée par des guerriers revêtus d'une armure dont les Mantinéens passaient pour être les inventeurs ${ }^{34}$.

D'après un témoignage de Platon, il ressort que les danses armées étaient rapportées, selon la région, à une divinité particulière que l'on estimait de la sorte imiter : ce sont, hormis les Courètes, les Dioskouroi chez les Lacédé-

28 PAUS., VIII, 37, 8. Cf. JOST, op. cit., p. 336.

29 É. LoucAs, Le nom de la Thea Despoina..., p. 405-408, 412-413. LoucAs \& LOUCASDURIE, La mention du mot «agalma»..., p. 572-578.

30 JEANMAIRE, op. cit., p. 430-431 et sq.

$31 I G, \mathrm{~V} 2,344$.

32 Cf. VIAN, op. cit., p. 239 et n. 3.

$33 I G, \mathrm{~V} 2$, 271. Cf. aussi G. FoUgères, Mantinée et l'Arcadie orientale, Paris, 1898 (BEFAR, 78), p. 297-298.

34 Cf. VIAN, op. cit., p. 243. Cf. aussi FougìRes, op. cit., p. 349-350. 
moniens et Athéna à Athènes ${ }^{35}$. La fête des Panathénées comportait en effet un concours de danseurs de pyrrhique ${ }^{36}$, danse (dont l'invention a été

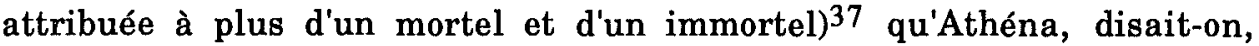
exécuta dès qu'elle sortit du crâne de Zeus ou après la destruction des Titans - à moins qu'il ne faille comprendre celle des Géants ${ }^{38}$.

Les Mantinéens, qui donnent l'impression d'avoir repris à leur compte la réputation de rudes guerriers dont jouissaient les Arcadiens déjà dans l'épopée homérique ${ }^{39}$, pratiquaient aussi l'hoplomachia et la monomachia qui comportaient des combats simulés, lesquels pouvaient parfois s'accompagner d'une orchestique ${ }^{40}$. On sait que si, lors de certaines célébrations particulières, les danses avaient un caractère libre et frénétique - comme dans le rituel en l'honneur de Despoina au "megaron" 41 -, à d'autres occasions elles étaient exécutées avec ou sans armes sur le rythme scandé par les instruments selon une chorégraphie définie, et qu'à ce titre elles tenaient une place importante dans l'éducation, particulièrement dans celle des jeunes gens se préparant à devenir citoyen ${ }^{42}$. Au dire de Polybe, qui souligne la place donnée à la musique et aux chants dans l'éducation en Arcadie, les jeunes gens s'exerçaient à marcher en rang au son de la flûte, s'entraînaient à des danses difficiles et, certains jours de l'année où étaient célébrés à grands frais des concours, se produisaient en public ${ }^{43}$. Selon $\mathrm{H}$. Jeanmaire, il s'agirait des apodeixeis que Plutarque (De Mus. 9, p. 1134) met sur le même rang que les airs accompagnant les Gymnopédies spartiates ${ }^{44}$.

Une autre des tribus de Mantinée, la tribu Enyalia, porte le nom d'une très ancienne divinité du combat, Ényalios ${ }^{45}$, particulièrement vénérée en Laconie et qui recevait à Sparte le sacrifice de deux chiots peu avant que deux équipes ne s'affrontent en un simulacre de combat au lieu-dit Platanistas ${ }^{46}$.

35 PLAT., Leg., VII, 796 b. Cf. aussi JEANMAIRE, op. cit., p. 428; É. LoUCAS, Le nom de la Thea Despoina..., p. 405, n. 31.

36 Cf., par ex., VIAN, op. cit., p. 249.

37 Cf. aperçu des sources chez L. SÉCHAN, La danse grecque antique, Paris, 1930, p. 9293.

38 LUCIEN, Dialogue des Dieux, 8 (226); DeN. HAL., VII, 72. Cf. VIAN, op. cit., p. 249.

39 HoM., Il., II, 604, 611; VIII, 134.

40 Xén., Anab., VI, 1, 4. VIAN, op. cit., p. 243; FoUGËRES, op. cit., p. 350-352.

41 Cf. LOUCAS \& LOUCAS-DURIE, La mention du mot «agalma»..., p. 573.

42 Plut., De Mus., 9, p. 1134. Cf. JeANMAIRE, op. cit., p. 441.

43 POLYB., IV, 20, 12; 28.

44 JEANMAIRE, op. cit., p. 441.

45 FoUGERES, op. cit., p. 294-296.

46 PAUS., III, 14, 6. 
Les protagonistes en étaient les «éphèbes» spartiates et ce drômenon, non dénué de caractère religieux, veillait à entretenir les qualités physiques exigées du chasseur pourvoyant à sa subsistance, du guerrier dont dépendait la sécurité et la prospérité de la citét ${ }^{47}$.

Les combats rituels s'inscrivent en effet dans un cadre général de renouvellement cyclique de la nature. La victoire des éléments bénéfiques étant périodiquement mise en jeu par les maléfiques, il est nécessaire de se l'assurer régulièrement. Et la condition même de l'existence des hommes et de toutes les créatures qui ne jouissent pas de l'éternité est de devoir continuellement lutter pour vivre et survivre, de mener un combat pour gagner la victoire qui assure la souveraineté, et pour la conserver ${ }^{48}$. De tels rites semblent avoir été représentés dans les lieux sacrés éleusiniens, par la ballètus, le lancer de pierres ${ }^{49}$, et le morotton, le combat à la massue ${ }^{50}$. Et des rites semblables existent même dans l'entourage de divinités devenues symbole du monde civilisé et de la nature domestiquée ${ }^{51}$.

Par Hérodote on apprend qu'une violente bataille entre deux camps de jeunes vierges qui se mesuraient à coups de pierres et de bâtons au péril de leur vie était périodiquement célébrée au bord du lac Tritonis en l'honneur de cette Athéna libyenne, fille de Poseidon, qu'adopta finalement Zeus ${ }^{52}$. Fr. Vian a également distingué des souvenirs de combat rituel, comme de sacrifice humain, dans certains motifs cultuels et mythiques de l'environnement de l'Athéna continentale ${ }^{53}$. Et dans les divers récits de ses enfances auprès d'un père nourricier, il est question d'une figure féminine, suntrophos de la déesse, qui trouve souvent la mort à la suite d'une rivalité

47 Sur les jeux spartiates et leur fonction à la fois religieuse et civique, cf. I. LoucAs, Пepi

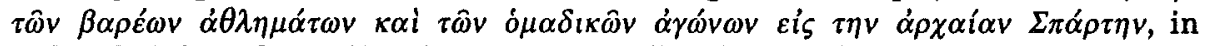
Lakonikai Spoudai, 7 (1983), p. 32-44; sur l'analogie à Sparte du chasseur et du guerrier, cf. P. VIDAL-NAQUET, «Le cru, l'enfant grec et le cuit», in Le chasseur noir, Paris, édit. rev. et corr. 1983, p. 177-207.

48 Cf. I. LOUCAS, art. cit., p. 39-40.

49 ATHen., IX, 406d; HesYch., s.v. Cf. L. FARNeLL, The Cults of the Greek States, III, Oxford, 1896, p. 93-94; G. MYLONAS, Eleusis and the Eleusinian Mysteries, Princeton, 1961 (1974), p. 140-141.

50 HESYCH., s.v.

51 Cf. le rôle de Déméter dans l'Hymne homérique à Déméter, tel qu'il a encore été dernièrement souligné par I. LOUCAS, Le comportement de la déesse de la prospérité...

52 HDT., IV, 180.

53 Cf. VIAN, op. cit., p. 270-271. 
survenant entre deux jeunes filles et donnant parfois lieu à un échange de coups relevant des exercices militaires ${ }^{54}$.

Fr. Vian a reconstitué un schéma analogue dans les enfances arcadiennes de Zeus, en combinant le témoignage des Arcadiens de Méthydrion, rapporté plus haut, et celui concernant le lieu-dit Bathos près de Trapézonte où, à proximité d'un culte des Grandes Déesses et de celui des Éclairs, de la Tempête et des Coups de Tonnerre, est localisée la Gigantomachie ${ }^{55}$ : élevé auprès d'Hoplodamos et ses Géants au mont Thaumasion, initié par eux au maniement des armes, Zeus affirmerait en foudroyant ses tuteurs insurgés qu'il est parvenu à sa majorité 56 .

Serait encore arcadien le thème qui apparente Athéna à l'ardeur combative et à la victoire, en faisant de Nikè, d'une part, la soeur de Zélos, Kratis et Biè, enfants de Pallas et de Styx, et de l'autre, la suntrophos de la déesse jusqu'à ce que celle-ci atteigne l'âge adulte - une relation dont on croit retrouver par ailleurs l'écho dans des cultes arcadiens, entre autres celui de Pallantion ${ }^{57}$.

Tous ces motifs cultuels et mythiques autour d'Athéna, Fr. Vian les reconnaît sous-jacents dans la Gigantomachie de la déesse et dans les jeux guerriers des Panathénées, fête devenue plus civique que religieuse ${ }^{58}$. A la manière dont elle entendait l'éducation civique et militaire, Athènes soumettait, elle aussi, ses éphèbes à des techniques de combat particulières, mais qui, en soulignant leur qualité de pré-hoplite, les situent, à ce premier stade de leur carrière militaire, aux frontières du monde civilisé incarné par la cite ${ }^{59}$.

L'éphébie athénienne était, à l'époque classique, de deux types : d'une part, l'éphébie officielle, d'un caractère purement civique et concernant la population masculine âgée de dix-huit ans, de l'autre, l'éphébie archaïque, correspondant à l'admission dans la phratrie à l'âge de seize ans, rite qui comprenait le sacrifice du koureion avec oblation de la coiffure, lors de la fête kouréôtis ${ }^{60}$. À chaque cité ses institutions. Si à Sparte, où les adultes avaient coutume de porter les cheveux longs, il ne semblait pas y avoir un net

54 Cf. VIAN, loc. cit..

55 PAUS., VIII, $29,1$.

56 VIAN, op. cit., p. 244-245.

57 DEN. HAL., I, 32; PAUS., VIII, 44, 5. Cf. VIAN, op. cit., p. 271.

58 VIAN., op. cit., ch. 3 et 4.

59 VIDAL-NAQUET, «Le chasseur noir et l'origine de l'éphébie athénienne», in Le chasseur noir, p. 173.

60 VIDAL-NAQUET, «L'origine de l'éphébie...», p. 155. 
passage de l'enfance à l'âge adulte ${ }^{61}$, à Athènes, la fin de l'adolescence se signalait par la consécration de la chevelure à Artémis ${ }^{62}$.

Selon les Arcadiens, Oreste fit de même sur la route qui conduit à Messène, en un lieu que rappelait le sanctuaire de Koureion. Cependant, la tradition ne rapporte pas ce geste à un rite ou à une coutume marquant le passage de l'adolescence à l'âge adulte : le héros, pris de folie à la suite du meurtre de sa mère, montrait de la sorte qu'il avait recouvré ses esprits ${ }^{63}$. Le geste d'Oreste se définit donc plutôt dans des termes d'abandon de l'état sauvage, signifié par la mania divine, en faveur de la civilisation.

Dans un passage de l'Hymne à Artémis se rapportant au contexte arcadien, Callimaque rappelait que Prö̈tos exprima sa gratitude à la déesse en lui consacrant deux temples, celui d'Hemera à Lousoi, pour avoir délivré ses filles de leur accès de folie, et un autre en tant que Koria, parce qu'elle avait d'abord regroupé les jeunes filles qui erraient, dispersées, de par les monts d'Azania ${ }^{64}$. Mais, selon Pausanias, Koria est l'épithète d'une autre divinité, tout aussi particulièrement associée aux kouroi et korai que l'est Artémis : Athéna, vénérée sur les hauteurs aux alentours de Kleitor ${ }^{65}$. La déesse était vraisemblablement associée à la fête célébrée à Kleitor sous le nom de Kor(i)asia ou Koreia et qui comportait des concours ${ }^{66}$. Or ces Kor(i)asia de Kleitor ont peut-être eu quelque rapport avec Lykosoura.

On a retrouvé à Lykosoura un décret des premières années de l'ère chrétienne par lequel la polis tôn Lukourasiôn remercie Nikasippos, prêtre de Despoina pour la deuxième fois consécutive, ainsi que sa femme, également prêtresse de Despoina lors de son second sacerdoce ${ }^{67}$. Nikasippos, apprend-on, n'avait pas hésité à prendre des responsabilités en des temps difficiles et avait fait preuve de largesses à différentes occasions au bénéfice du culte. Il s'inscrit ainsi dans la ligne de ses ancêtres, qui ont rempli avec

61 HDT., I, 82; PLUT., Lyc., 1. Cf. VIDAL-NAQUET, «Le cru, l'enfant grec et le cuit...”, p. 203204.

62 HeSYCH, s.v. коupeânıs.

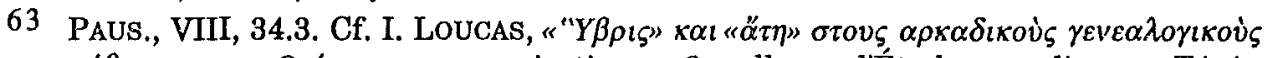

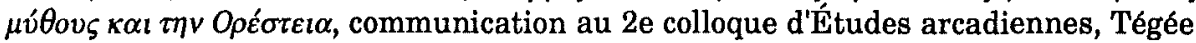
- Tripolis, 11-4/11/1988 (actes sous presse).

64 Callim., Hymn. Dian., 233-236.

65 PAUS., VIII, 21, 4.

66 Inscription de Tralles (fragment d'un décret honorifique du temps des Séleucides) in $B C H, 10$ (1886), p. 326-327; IG, VII, 47; Schol. PIND., Ol., VII, 153. Cf. M.P. NILsson, Griechische Feste, Leipzig, 1906, p. 91; R. STIGLITZ, Die Grossen Gottinnen Arkadiens, Vienne, 1967 (Sonderschr. Esterr. Archäol. Inst. in Wien, XV), p. 100.

$67 I G, \mathrm{~V} 2,516=S y l l .{ }^{3}, 800=S E G, 14$ (1957). Cf. É. (LoUCAS-) DURIE, Les fonctions sacerdotales..., p. 138-141. 
assiduité et magnanimité leurs devoirs envers la cité comme envers les dieux dans les sundikiaia, les sacerdoces et les teknôn Koreitiai, entre autres dépenses.

Démunis de tout renseignement supplémentaire, les chercheurs ont parfois expliqué ces Koreities comme une célébration en l'honneur de Corè (Perséphone) - dont la présence à Lykosoura serait, de cette manière, pour la seule fois suggérée 68 . Mais on a pensé aussi à l'éventualité d'une relation, tout au moins à l'origine, avec les Kor(i)asia ou Koreia à Kleitor ${ }^{69}$.

Et si, dans le contexte cultuel de Lykosoura, Artémis - dont l'effigie faisait pendant à celle d'Anytos dans le groupe sculpté - a gagné une place primordiale ${ }^{70}$, Athéna n'a pas été ignorée. Au-delà du sanctuaire de Despoina, dit Pausanias, Athéna était honorée par un hieron aménagé en plus d'un xoanon ${ }^{71}$. Et parmi les plus anciens objets provenant du sanctuaire même de la Souveraine des lieux, on compte une statuette archaïque en bronze d'Athéna du type promachos et quelques ex-voto contemporains, découverts dans cette chambre carrée qui jouxte l'extrémité occidentale du portique et "dont la destination n'est pas claire» 72 . Une autre statuette archaïque en bronze figurant une Athéna combattant proviendrait également de Lykosoura, de même qu'une statuette d'hoplite cuirassé et armé ${ }^{73}$.

Si cette image vous rappelle Anytos, sachez qu'au contraire de cet hoplite coiffé d'un casque de type corinthien à haut cimier recourbé en avant, le parèdre de Despoina a lui la tête nue, et ses cheveux hirsutes se mêlent aux boucles de sa barbe.

Thisseos, 45

Éveline LOUCAS-DURIE

Chalandri

GR - 15234 ATHÈNES

68 H.G. LIDDELL - R. SCOTT - H.S. JONES, A Greek-English Lexikon, Oxford, 1968, p. 980, s.v. Kopєıı́ $\alpha$; ChantraIne, Dictionnaire..., II, E-K, Paris, 1970, p. 567, s.v. кópos. Cf. JosT, op. cit., p. 334, n. 1.

69 Cf. l'annotation 4 du commentaire du décret de Lykosoura en l'honneur de Nikasippos ( ${ }^{\circ} 34$, p. 316-322, plus spécialement p. 321) fait par $H$. Taeuber dans le cadre de la préparation d'une publication d'inscriptions grecques à aspect juridique par l'Université de Vienne, aimablement communiquée par l'auteur.

70 Cf. É. LoucAs, Le nom de la Thea Despoina...; LoucAs \& LoucAs-DuRIE, La mention du mot «agalma»..., p. 572 sq.

71 PAUs., VIII, 37, 10.

72 Jost, op. cit., p. 173. Cf. aussi, du même auteur, Statuettes de bronze archaïques provenant de Lykosoura, in BCH, 99 (1975), p. 339-364, plus spécialement p. 352355.

73 Athènes, MN 12736 et Mn 7644. Cf. JosT, Statuettes de bronze... 